OPEN ACCESS

Edited by:

Lei Wang,

Ocean University of China, China

Reviewed by:

Enmin Zong,

Taizhou University, China

Xin Wang,

Nanjing Tech University, China

*Correspondence:

Jifu Wang

wif118@126.com

Fuxiang Chu

chufuxiang@caf.ac.cn

Specialty section:

This article was submitted to

Bioprocess Engineering,

a section of the journal

Frontiers in Bioengineering and

Biotechnology

Received: 05 August 2021 Accepted: 20 September 2021 Published: 02 November 2021

Citation:

Yu J, Xu C, Lu C, Liu Q, Wang J and

Chu $F$ (2021) Synthesis of $p H$-Sensitive and Self-Fluorescent Polymeric Micelles Derived From Rosin and

Vegetable Oils via ATRP.

Front. Bioeng. Biotechnol. 9:753808.

doi: 10.3389/fbioe.2021.753808

\section{Synthesis of $\mathrm{pH}-$ Sensitive and Self-Fluorescent Polymeric Micelles Derived From Rosin and Vegetable Oils via ATRP}

\author{
Juan $Y u^{1,2}$, Chaoqun $X u^{1}$, Chuanwei Lu ${ }^{1,2}$, Qian $L_{i u^{3}}$, Jifu Wang ${ }^{2 *}$ and Fuxiang Chu ${ }^{1,2 *}$ \\ ${ }^{1}$ Key Laboratory of Forestry Genetics and Biotechnology, Jiangsu Province Key Laboratory of Green Biomass-based Fuels and \\ Chemicals, Ministry of Education, Jiangsu Co-Innovation Center of Efficient Processing and Utilization of Forest Resources, \\ College of Chemical Engineering, Nanjing Forestry University, Nanjing, China, ${ }^{2}$ National Engineering Laboratory of Biomass \\ Chemical Utilization, Key and Laboratory of Forest Chemical Engineering, SFA, Key Laboratory of Biomass Energy and Material, \\ Institute of Chemical Industry of Forest Products, CAF, Nanjing, China, ${ }^{3}$ Research Center for Nanophotonic and Nanoelectronic \\ Materials, School of Materials Science and Engineering, Suzhou University of Science and Technology, Suzhou, China
}

Preparation and application of sustainable polymers derived from renewable resources are of great significance. The aim of this study is to synthesize a kind of sustainable polymeric micelles from rosin and vegetable oils via atom transfer radical polymerization (ATRP) and to investigate the doxorubicin delivery properties of these micelles. Dehydroabietic acid-based poly lauryl methacrylate (DA-PLMA) with narrow PDI of 1.13 was prepared in a well-controlled process using rosin as an ATRP initiator. Thereafter, carboxylic groups were introduced to form poly methacrylic acid (PMAA) moieties in DA-PLMA polymer via acid hydrolysis. The resulted DA-PLMA-PMAA could self-assemble in water to form $\mathrm{pH}$ dependent polymeric micelles with a diameter of $\sim 65 \mathrm{~nm}$ and PDI as low as 0.105. Owing to the existence of rosin, DA-PLMA-PMAA micelles also showed self-fluorescence properties. In addition, Dox-loaded micelles were prepared in aqueous solution with the drug-loading capacity as high as $16.0 \%$ and showed sustained-release characteristics. These results demonstrate great promise for designing polymeric micellar from rosin and vegetable oils.

Keywords: pH-sensitive, self-fluorescence, polymeric micelles, rosin, vegetable oils, ATRP, doxorubicin

\section{INTRODUCTION}

The application of renewable raw materials (included natural polymers and chemicals) can take advantage of the synthetic potential of nature and avoid or minimize $\mathrm{CO}_{2}$ emission, which is of great significance for carbon emissions and carbon neutralization (Meier et al., 2007; Thyavihalli Girijappa et al., 2019). Renewable natural polymers such as cellulose, hemicellulose, starch, chitin, lignin, and natural rubber have been widely explored in chemical industry for the partial replacement of fossil fuel-based polymeric materials (Biermann et al., 2011; Zhang et al., 2017; Wang J. et al., 2020; Sun et al., 2020). However, natural rosin, a kind of important natural chemicals, which can be easily obtained from pine resins and some other related plants, has been modified to be various kinds of fine chemicals and monomers to replace petrochemicals in industry (Cai et al., 2021). These rosin-based monomers could be polymerized via controlled radical polymerization resulting in a class of welldefined rosin-derived polymers. Owing to the good thermal properties, excellent hydrophobicity, 


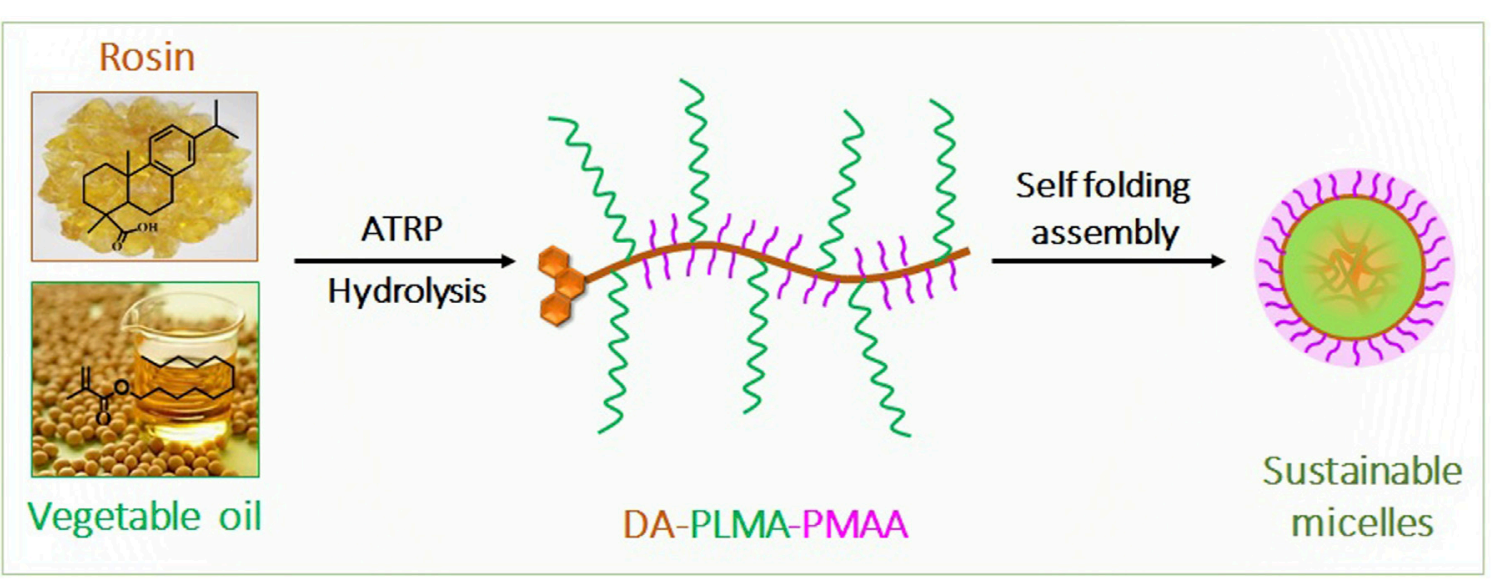

SCHEME 1 | Schematic illustration of synthesis of DA-PLMA-PMAA sustainable micelles from rosin and vegetable oil.

biocompatibility, and UV absorption properties, these renewable rosin-derived polymers could be potential candidates for thermoplastic elastomers. Recently, rosin was transferred to an atom transfer radical polymerization (ATRP) initiator which provides an alternative avenue for the designation of rosinbased polymers (with rosin content less than $10 \mathrm{wt} \%$ ) to achieve the improvement in thermal, UV-blocking, and mechanical performance (Yu et al., 2021).

Polymeric micelles formed by self-assembly of amphiphilic block copolymers have been widely applied as excellent carriers for hydrophobic drugs because they have high drug-loading capacity, sustained release manner (owing to their hydrophobic core), and the stabilization ability in aqueous solution (owing to their hydrophilic shell) (Tyrrell et al., 2010; Tang et al., 2011; Bastakoti et al., 2013; Sun et al., 2018). Therefore, a number of amphiphilic block copolymers have been synthesized and reported to form a hydrophilic shell of micelles in aqueous solution. Regarding amphiphilic block copolymers for polymeric micelles, PEG is the most widely used hydrophilic block; the commonly reported hydrophobic blocks are poly (propylene oxide) (PPO), poly ( $\varepsilon$-caprolactone) (PCL) and poly (lactide) (PLA), and poly lauryl methacrylate (PLMA) (Tyrrell et al., 2010; Tang et al., 2011; Hattori et al., 2017). There are various method choices for the synthesis of amphiphilic block copolymers, including free radical polymerization (Debele et al., 2017), ring-opening polymerization (Dai et al., 2011), reversible addition-fragmentation chain transfer (RAFT) (Wang C. et al., 2020), and ATRP (Oh et al., 2007).

However, most of the reported polymeric micelles were prepared from fossil fuel-based polymers. Research works focusing on polymeric micelles from renewable resource were rarely reported. Hespel et al. (2012) reported the synthesis of pHsensitive micelles from renewable linseed oil via ATRP. The amphiphilic copolymers from linseed oils were prepared by ATRP of tert-butyl acrylate (tBA) from a linseed oil initiator and subsequent acidolysis of the PtBA block into poly(acrylic acid) (PAA). Compared to amphiphilic block copolymers, the preparation of amphiphilic random copolymers is much easier. In addition, it is reported that amphiphilic copolymers obtained by copolymerizing a hydrophilic poly(ethylene glycol) (PEG) monomer and a hydrophobic lauryl monomer with a random monomer sequence could produce uniform micelles in water, and the micelle's size was determined by the composition (Hattori et al., 2017). These results promote the fabrication of polymeric micelles derived from renewable vegetable oil resources.

The objective of this study was to develop polymeric micelles based on rosin and vegetable oils as a drug carrier and to reveal the role of rosin in polymeric micelles. As shown in Scheme 1, a rosin-based initiator (2-BriBEDA) and lauryl methacrylate (LMA, derived from vegetable oil) were used as raw materials. Hydrophobic dehydroabietic acid-based poly (lauryl methacrylate) (DA-PLMA) was prepared via ATRP using 2BriBEDA as an initiator. After acid hydrolysis, poly methacrylic acid (PMAA) moieties were introduced in PLMA blocks, resulting in an amphiphilic random copolymer (DAPLMA-PMAA). The self-assembly of DA-PLMA-PMAA into polymeric micelles was verified and characterized. Hydrophobic drug doxorubicin (Dox) was encapsulated into the resulted micelles. This novel type of biomass-based polymeric micelles exhibit $\mathrm{pH}$-sensitivity, self-fluorescent, high drug-loading level capacity, and aqueous stability, making it an extremely promising nanoplatform for the drug carrier field.

\section{EXPERIMENTAL}

\section{Materials}

A rosin-based ATRP initiator (2-BriBEDA) was prepared using dehydroabietic acid (DA, main content of rosin) as a raw material according to our previous study (Yu et al., 2021). N,N, $N^{\prime}, N^{\prime \prime}, N^{\prime \prime}$ pentamethyldiethylenetriamine (PMDETA, 99\%, Aladdin Industrial Inc.), lauryl methacrylate (LMA, 96\%, Aldrich), and $\mathrm{CuBr}$ (99.999\%, Aldrich) were used as received. Doxorubicin hydrochloride (Dox $\mathrm{HCl}$ ) was purchased from Beijing Huafeng United Technology Company. Tetrahydrofuran (THF, AR 


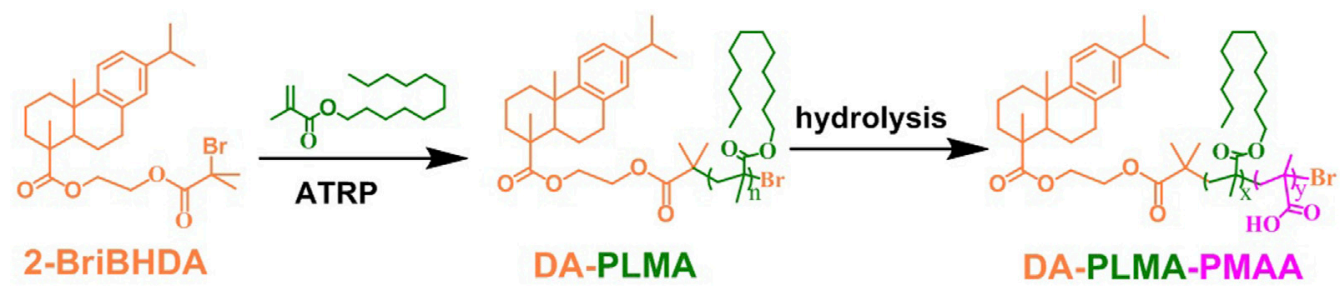

FIGURE 1 | Schematic illustration of synthesis of DA-PLMA from DA-based initiators, and partial hydrolysis of DA-PLMA to prepare DA-PLMA-PMAA.

reagents), dichloromethane (DCM, AR reagents), methanol (AR reagents), and petroleum ether (AR reagents) were purchased from Nanjing Reagent Chemical Co. Ltd. Anisole was dried over $4 \mathrm{~A}$ molecular sieves and then distilled before use.

\section{Characterization}

An FT-IR analysis of rosin-based polymers before and after acid hydrolysis was performed using a Nicolet iS10 FT-IR spectrometer by an attenuated total reflectance method.

A ${ }^{1} \mathrm{H}$ NMR analysis of rosin-based polymers before and after acid hydrolysis was carried out on a Bruker DMX 300 NMR spectrometer, and $\mathrm{CDCl}_{3}$ or DMSO- $\mathrm{d}_{6}$ was used as the solvent.

Gel permeation chromatography (GPC) was performed at $40^{\circ} \mathrm{C}$ to measure molecular weight and molecular weight distribution of rosin-based polymers. All samples were filtered over a microfilter with a pore size of $0.22 \mu \mathrm{m}$ (Nylon, Millex-HN $13 \mathrm{~mm}$ Syringes Filters, Millipore). A Malvern Viscotek 3580 System, a Viscotek GPC2502 refractive detector, and a GPC1007 pump were involved. An HPLC-grade THF was used as the eluent, and the flow rate was $1 \mathrm{ml} / \mathrm{min}$. Monodispersed polystyrene (PS) was used as the standard to generate the calibration curve.

A UV-visible spectrophotometer (UV/vis) analysis was performed by recording the solution of rosin-based polymers before and after acid hydrolysis in THF on a Shimadzu UV-1800, Shimadzu Scientific Instruments Incorporated in a double-beam mode, whereas pure THF was used as a reference of the copolymer solution while the wavelength is between 200 and $400 \mathrm{~nm}$.

Fluorescence (FL) spectra were used to characterize the fluorescence properties, which were measured on a HITACHI F-4500 spectrofluorometer with the band widths of $10 \mathrm{~nm}$ for excitation and $2.5 \mathrm{~nm}$ for emission, where the excitation wavelength (lex) was $360 \mathrm{~nm}$. The samples were dissolved in THF with a concentration of $2 \mathrm{mg} / \mathrm{ml}$.

Surface tension of the micelle's solution was measured by using the Wilhelmy plate technique at $25^{\circ} \mathrm{C}$. The tensiometer was calibrated against water before measurements. The platinum plate was always cleaned and heated to red color with an alcohol lamp.

Transmission electron microscope (TEM) images were obtained by a JEOL JEM-2011 instrument operated at $100 \mathrm{kV}$. For TEM studies, a drop of micellar solution was deposited on an electron microscopy copper grid coated with a carbon film, and the water was evaporated at room temperature.
The sizes, size distributions, and zeta-potentials of prepared micelles were determined using a Zetasizer Nano-ZS Instrument (ZEM4228, Malvern Instruments, United Kingdom). Each sample was equilibrated at $25^{\circ} \mathrm{C}$ for $1 \mathrm{~min}$ before measurement, and five sets of time-averaged measurements were taken. A $633 \mathrm{~nm} \mathrm{He-Ne} \mathrm{laser} \mathrm{was} \mathrm{the} \mathrm{light} \mathrm{source.}$

\section{Synthesis of DA-PLMA via ATRP}

2-BriBHDA was used as the initiator to initiate the polymerization of vegetable oil-based monomer lauryl methacrylate (LMA) via ATRP. As shown in Figure 1, for a typical polymerization procedure, a mixture of LMA (2.57 g, 1.01 $\left.\times 10^{-2} \mathrm{~mol}\right)$, PMDETA $\left(9.13 \mathrm{mg}, 5.07 \times 10^{-5} \mathrm{~mol}\right), 2$-BriBHDA $\left(25 \mathrm{mg}, 5.07 \times 10^{-5} \mathrm{~mol}\right)$, and anisole $(3 \mathrm{ml})$ was charged into a round-bottom flask and degassed by three freeze-pump-thaw cycles. Thereafter, the mixture was transferred into a Schlenk flask that contained $\mathrm{Cu}(\mathrm{I}) \mathrm{Br}\left(7.5 \mathrm{mg}, 5.07 \times 10^{-5} \mathrm{~mol}\right)$ under nitrogen and soon placed in an oil bath set at $100^{\circ} \mathrm{C}$. Periodically, samples were withdrawn from the Schlenk flask via a syringe under nitrogen to determine the monomer conversion, molecular weight, and polydispersity of the resulted polymer. The polymerization was stopped by pouring the reaction mixture with THF. After passing through an alkaline alumina column, the product was precipitated into methanol, collected, and dried under vacuum until constant weight.

\section{Preparation of DA-PLMA-PMAA by Acid Hydrolysis of DA-PLMA}

$1.5 \mathrm{ml}$ of concentrated sulfuric acid was added to a Teflon centrifugal tube containing $100 \mathrm{mg}$ DA-PLMA copolymer; subsequently, the reaction mixture was stirred in $45^{\circ} \mathrm{C}$ water bath for $12 \mathrm{~h}$. DA-PLMA-PMAA were obtained by filtration and washed with a plenty of water/methanol mixture prior to being lyophilized (Semen, 1969).

\section{Micelle Formation}

Briefly, the DA-PLMA-PMAA copolymer (10 mg) was dissolved in $2 \mathrm{ml}$ THF to give $5 \mathrm{mg} / \mathrm{ml}$ solutions, followed by the dropwise addition into distilled water with vigorous stirring until the concentration reached to $0.1 \mathrm{mg} / \mathrm{ml}$. The mixture was left to stand at room temperature for $24 \mathrm{~h}$ and was filtered over a microfilter with a pore size of $0.45 \mu \mathrm{m}$ prior to the subsequent measurement. The polymeric micelle with concentration of $1 \mathrm{mg} /$ $\mathrm{ml}$ was prepared in a similar way. 
A

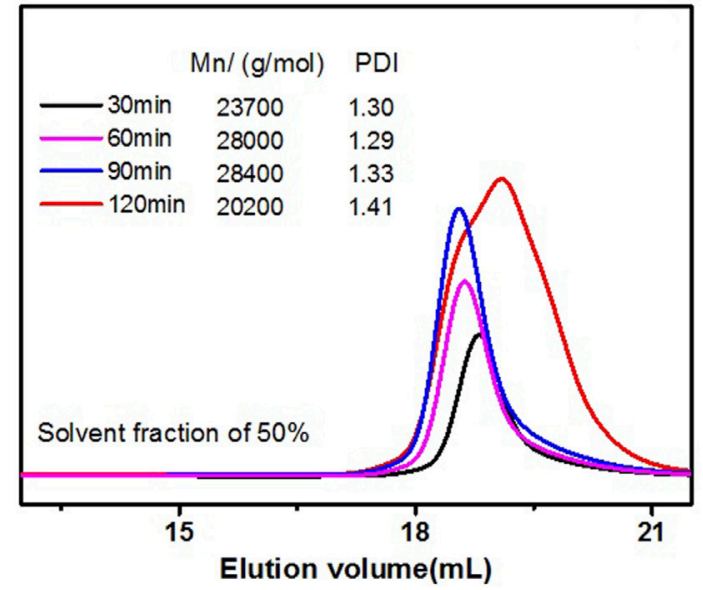

B

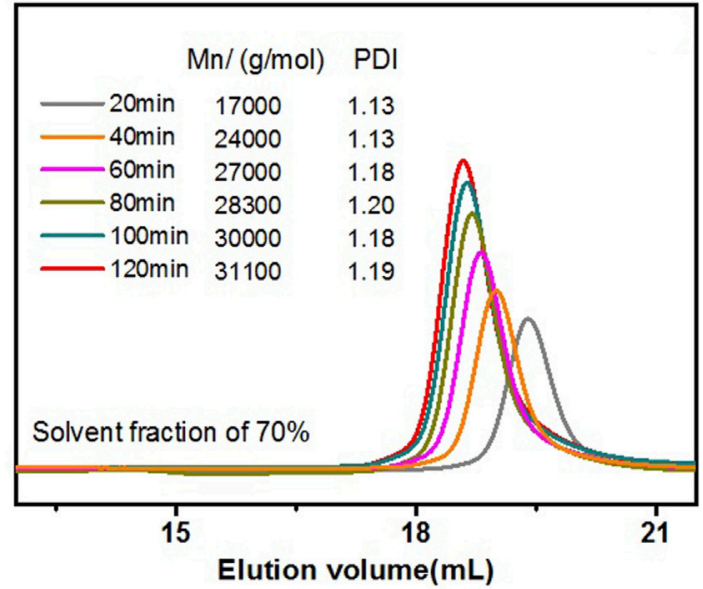

FIGURE 2 | GPC traces of DA-PLMA polymers and Mn/PDI using anisole as a solvent with different reaction times: (A) solvent fraction of 50\% and (B) solvent fraction of $70 \%$.

\section{Preparation of Dox-Loaded Micelles and In Vitro Drug Release}

Dox-loaded micelles were prepared by an incubation method according to our previous study (Nan et al., 2014). The micelle solution (prepared above) was mixed with Dox solution of various predetermined concentrations at room temperature. Subsequently, in order to allow the DOX/micelle mixture to reach an equilibrium state, the mixed solution was incubated at $37^{\circ} \mathrm{C}$ for $12 \mathrm{~h}$. To determine the entrapment efficiency (EE) and drug-loading efficiency (LE), the drug-loaded micelle solution was centrifuged, and the amount of free Dox in the solution was analyzed by using a UV-vis spectrometer at $495 \mathrm{~nm}$, using a standard calibration curve experimentally obtained with DOX/ water solution. The drug-loading capacity (LC) and drug-loading efficiency (LE) were calculated according to the following formulas:

$\mathrm{LE}(\%)=$ Weight of DOX Loaded in micelles/Weight of feeding DOX $* 100 \%$,

$\mathrm{LC}(\%)=$ Weight of DOX Loaded in micelles $/$ Weight of micelles $* 100 \%$.

The centrifuged DOX/micelle mixture was dispersed in water and then loaded into a dialysis bag (molecular weight cut-off: $14 \mathrm{kDa})$ and dialyzed against $\mathrm{PBS}(\mathrm{pH}=7.4)$ in a beaker at $37^{\circ} \mathrm{C}$ for in vitro drug release. At selective time intervals, $3 \mathrm{ml}$ of solution was withdrawn from the release medium and replaced with $3 \mathrm{ml}$ fresh PBS. The DOX content in the samples was analyzed using the UVvis spectrophotometer at $495 \mathrm{~nm}$.

\section{RESULTS AND DISCUSSION}

\section{Synthesis of DA-PLMA via ATRP}

Given that the polydispersion index (PDI) of the polymer has a key effect on the PDI of polymeric micelles, the ATRP polymerization condition of DA-PLMA was optimized first to obtain narrow PDI for the polymers. As shown that $\mathrm{PMDETA} / \mathrm{CuBr}$ is the best catalyst system for the rosinbased ATRP initiator (Yu et al., 2021), the influence of solvents was emphasized in this study. It is reported that for the polymerization of PLMA, the PDI of PLMA obtained in anisole was a little narrower than those obtained in the other investigated solvents (DMF, acetonitrile, benzene, or toluene) (Çayli and Meier, 2008). Therefore, anisole was chosen as the solvent in this condition. Two different solvent fractions were employed, respectively, with the same feeding ratio $\{[\mathrm{LMA}] /[\mathrm{I}] /$ $[\mathrm{Cu}(\mathrm{I})] / \mathrm{PMDETA}=200 / 1 / 1 / 1\}$ as solvent fraction could affect ATRP equilibrium constants ( $\mathrm{K}_{\mathrm{ATRP}}$ ) (Wang et al., 2012). Commonly, the lower $\mathrm{K}_{\mathrm{ATRP}}$ may lead to a better controlled polymerization. The results of $\mathrm{Mn}$ as well as PDI were monitored by GPC. As shown in Figure 2A, the GPC traces of DA-PLMA were symmetrical and monomodal peaks at first $60 \mathrm{~min}$, but became wider after $60 \mathrm{~min}$ with a decrease of $\mathrm{Mn}$ and increase of PDI. The final products obtained at $120 \mathrm{~min}$ had a Mn of $20,200 \mathrm{~g} / \mathrm{mol}$ and PDI of 1.41 , which means that the ATRP polymerization of LMA was not controlled well in this condition. However, when the solvent fraction increased to $70 \%$, all the GPC traces at different reaction times (from 20 to $120 \mathrm{~min}$ ) showed symmetrical and monomodal peaks, Mn values changed linearly with the reaction time, and all the PDI values were not more than 1.2, confirming the controlled character of polymerization (Figure 2B). It should be noted that the reaction medium is initially a mixture of solvent/monomer, but it gradually changed to solvent/ monomer/polymer as the polymerization proceeds. The polymer fraction increased with increasing reaction time, and so was the viscosity of the reaction medium, especially in the reaction with lower solvent fraction. The reason why higher solvent fraction leads to a better control of polymerization in this study is probably because the viscosity of the reaction medium was not increased so much that affects the 

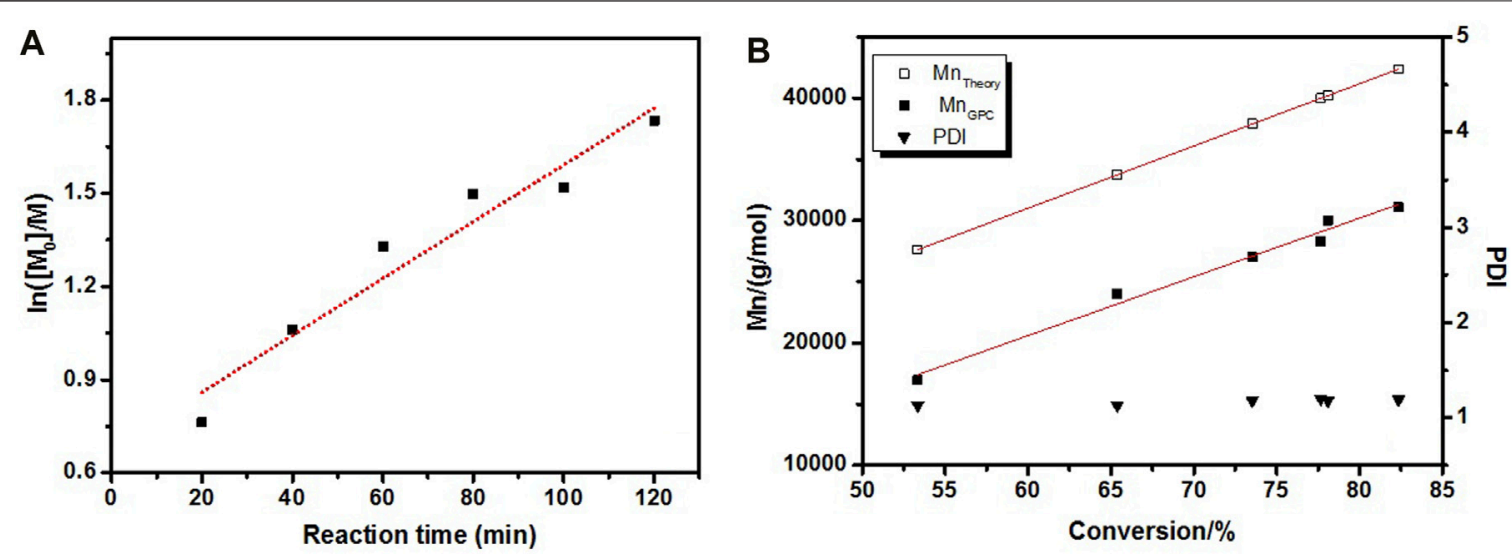

FIGURE 3 | Kinetic plots for the polymerization of LMA (A) and evolution of the $M_{\mathrm{n}}$ (theoretical and GPC) and PDI (B).

polymerization. Thus, anisole (volume 70\%) was used as the solvent in most cases.

The controlled and living nature of polymerizations were verified through kinetic experiment which was monitored by analyzing the reaction mixture with ${ }^{1} \mathrm{H}$ NMR spectroscopy and GPC. The monomer conversion was calculated via the ${ }^{1} \mathrm{H}$ NMR spectrum of the reaction mixture according to Eq. 3 and GPC given Mn and PDI of resulted polymer. The semilogarithmic plot of monomer conversion vs. reaction time is shown in Figure 3A. The linear dependence of $\ln \left([\mathrm{M}]_{0} /[\mathrm{M}]\right)$ on time suggested that the polymerization is a well-controlled process. The $M_{n}$ s obtained by GPC is shown in Figure 3B, and the molecular weight of copolymers increased with conversion. Meanwhile, the PDI remained less than 1.2 over the whole reaction, meaning a good control of the final copolymer composition (Hespel et al., 2012). In addition, the number average molecular weights of the copolymers determined by GPC were not in fair agreement with the theoretical values calculated by ${ }^{1} \mathrm{H}$ NMR (Figure 3B). The lower molecular weights determined by GPC could be explained by a notion that molecular weights determined by GPC using PS standards correspond to PS-equivalent molecular weights (Netopilı' $\mathrm{k}$ and Kratochvi' 1 , 2003). Therefore, it is the hydrodynamic volume disparity between PS and PLMA that leads to the discrepancy between theoretical and experimental $M_{n}$ s.

$$
\text { LMA conversion }(\%)=\left(1-18 / \mathrm{A}_{1.31} * \mathrm{~A}_{5.54}\right) * 100 \% \text {, }
$$

where $\mathrm{A}_{1.31}$ is the ${ }^{1} \mathrm{H}$ NMR integration area $(\sim 1.31 \mathrm{ppm})$ of methylene protons in the lauryl of LMA, $\mathrm{A}_{5.54}$ is the ${ }^{1} \mathrm{H}$ NMR integration area ( $5.54 \mathrm{ppm})$ of unsaturated protons in LMA, and 18 is the number of protons of methylene protons in the lauryl of LMA.

\section{Chemical Structure of DA-PLMA and DA-PLMA-PMAA}

Amphiphilic copolymers with both hydrophobic segments and hydrophilic segments could autonomously self-assemble to be micelles or vesicles in water via hydrophobic effects (Hattori et al., 2017). Therefore, DA-PLMA-PMAA was obtained by acid hydrolysis of the hydrophobic polymer DA-PLMA. During acid hydrolysis, hydrophobic lauryl was removed while the hydrophilic carboxyl group was introduced at the same time. ATR-FT-IR, ${ }^{1} \mathrm{H} \mathrm{NMR}$, the UV/vis analysis, and GPC were employed to characterize the chemical structure of DA-PLMA before and after hydrolysis.

The ATR-FT-IR analysis (Figure 4A) of DA-PLMA showed the appearance of $\mathrm{C}=\mathrm{O}$ signal peak at $1730 \mathrm{~cm}^{-1}$ as well as the peak of C-O-C at $1,240 \mathrm{~cm}^{-1}$ which ascribed to ester group in PLMA parts. The absorption peaks at around $720 \mathrm{~cm}^{-1}$ and $750 \mathrm{~cm}^{-1}$ correspond to the $-\mathrm{CH}_{2}$ group in the side lauryl chain of PLMA and the main chain of PLMA, respectively, while the appearance of absorption peaks at around $3,100 \mathrm{~cm}^{-1}(\mathrm{O}-\mathrm{H}), 2,600 \mathrm{~cm}^{-1}(\mathrm{O}-\mathrm{H})$, and $1,700 \mathrm{~cm}^{-1}(\mathrm{C}=\mathrm{O})$ in the spectra of hydrolysis polymer (DA-PLMA-PMAA) indicated the formation of carboxylic groups after hydrolysis; however, the peak at $1,730 \mathrm{~cm}^{-1}$ and $720 \mathrm{~cm}^{-1}$ still exist meant that the partial hydrolysis of DA-PLMA and the hydrophobic lauryl side chain was also retained in the resulted DA-PLMAPMAA. The ${ }^{1} \mathrm{H}$ NMR analysis (Figure 4B) showed the appearance of peak at $12.2 \mathrm{ppm}$ corresponding to the protons of $\mathrm{O}-\mathrm{H}$ moiety in carboxylic groups which verifies the successful introduction of carboxylic groups by acid hydrolysis. The $-\mathrm{OCH}_{2}$ protons of PLMA (3.9 ppm) and $-\mathrm{CH}_{2}$ protons $(\sim 1.31 \mathrm{ppm})$ in lauryl side chains of LMA still present in the ${ }^{1} \mathrm{H}$ NMR spectrum of DA-PLMA-PMAA, further confirming the partial hydrolysis of DA-PLMA. The absence of the protons of rosin (usually around 6.8-7.3 ppm) probably caused the entanglement of lauryl long side chains of PLMA around the rosin-based initiator, which could be seen by zooming in the ${ }^{1} \mathrm{H}$ NMR spectrum (Yu et al., 2021).

As rosin and its derivatives have an absorption in the UV region (200-400 nm), the UV/vis analysis was further employed to confirm the existence of rosin in DA-PLMA and DA-PLMAPMAA. As shown in Figure 5A, the rosin-based initiator (2BriBHDA, $0.33 \mathrm{mg} / \mathrm{ml}$ ) had a UV absorption peak around $250-280 \mathrm{~nm}$. The obvious absorption around $250-280 \mathrm{~nm}$ was 

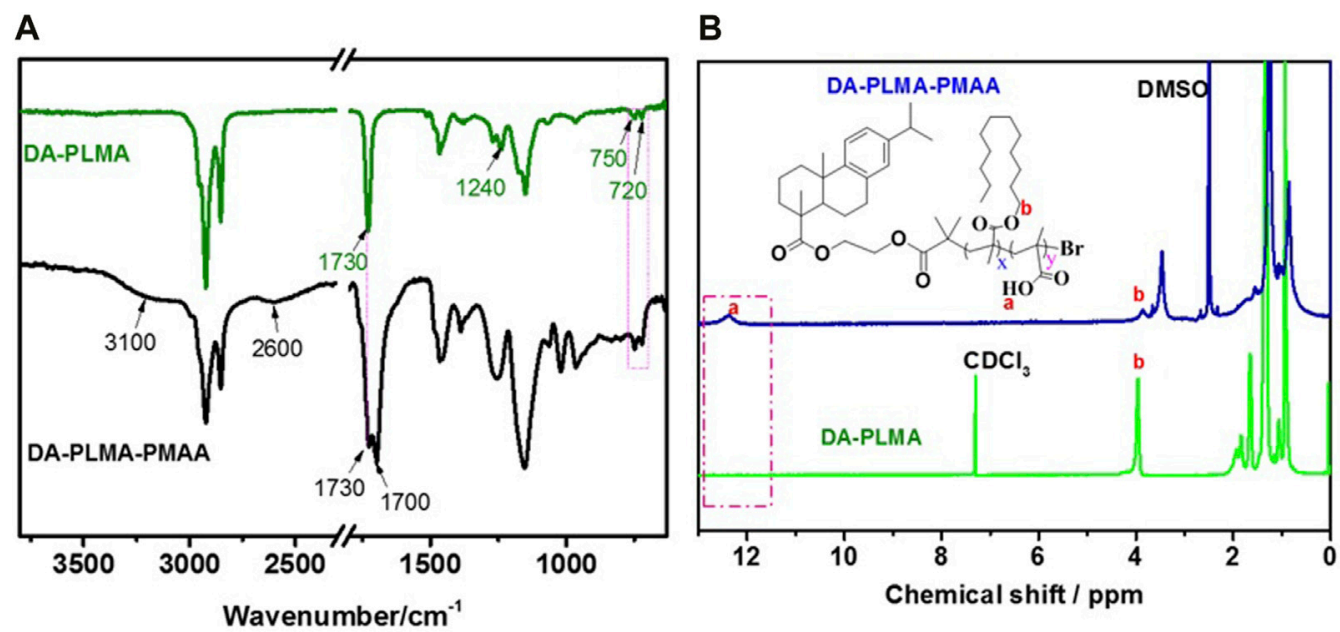

FIGURE 4 | Spectrum of DA-PLMA before and after hydrolysis (A) FT-IR and (B) ${ }^{1} \mathrm{H}$ NMR.
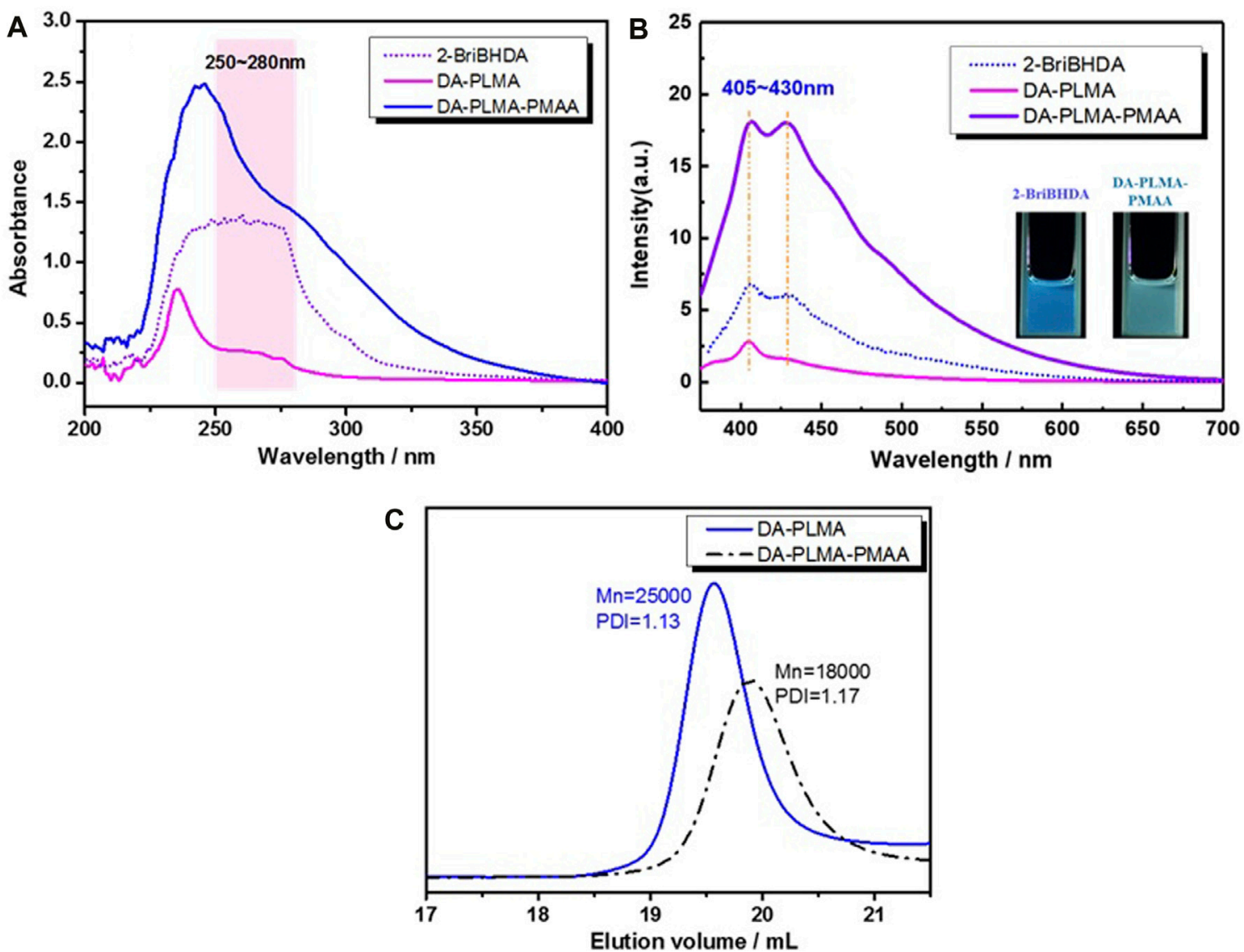

FIGURE 5 | (A) UV absorption curves and (B) fluorescence spectra (inset: digital photograph taken under $365 \mathrm{~nm}$ ) of the rosin-based initiator, DA-PLMA, and DAPLMA-PMAA in THF; (C) GPC traces of DA-PLMA and DA-PLMA-PMAA. 

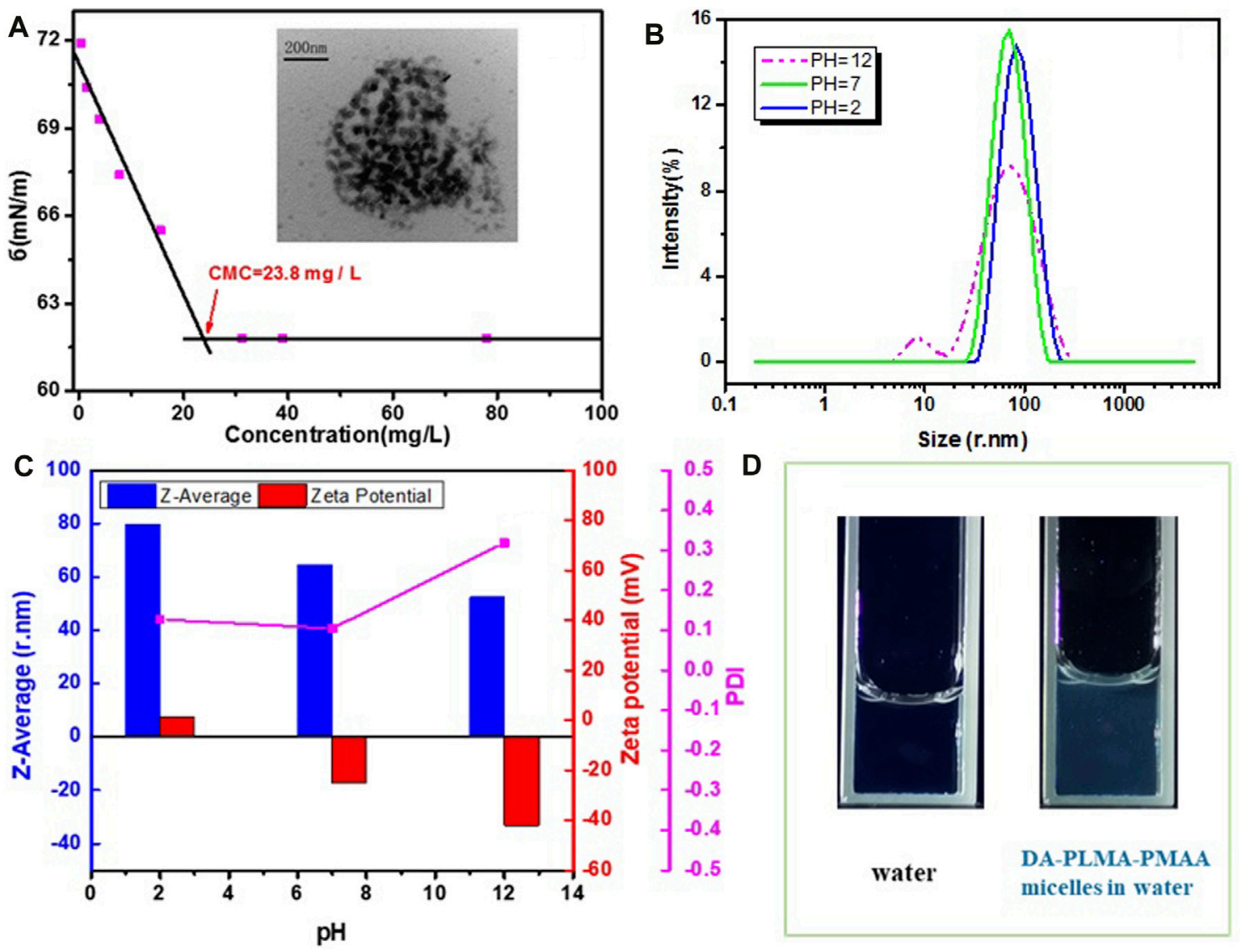

FIGURE 6 | (A) TEM image of micelles (inset image) and surface tension vs. micelle concentration at pH = 7; (B) size distribution profiles by intensity of micelles in different pHs; (C) Z-average, PDI, and zeta potential of micelles in different pHs; and (D) fluorescence spectra of water and DA-PLMA-PMAA micelles in water (digital photograph taken under $365 \mathrm{~nm}$ ).

clearly presented in the UV spectrum of DA-PLMA $(2 \mathrm{mg} / \mathrm{ml})$. After acid hydrolysis, UV absorption intensity around $250-280 \mathrm{~nm}$ increased significantly for DA-PLMA-PMAA $(2 \mathrm{mg} / \mathrm{ml})$. This is caused by the fact that the DA molar concentration increased after partial hydrolysis of DA-PLMA under the same mass concentration. As shown in Figure 5B (inset), the rosin-based ATRP initiator 2-BriBHDA $(10 \mathrm{mg} / \mathrm{ml})$ displayed the fluorescence in THF when excited at $365 \mathrm{~nm}$, so was DA-PLMA-PMAA (1 mg/ml). Therefore, the fluorescent emission spectra of 2-BriBHDA, DA-PLMA $(4 \mathrm{mg} / \mathrm{ml})$, and DA-PLMA-PMAA $(1 \mathrm{mg} / \mathrm{ml})$ in THF $\left(\lambda_{\text {ex }}=365 \mathrm{~nm}\right)$ were recorded to verify the role of rosin in regard to the fluorescence property (Figure 5B). 2-BriBHDA has a $\lambda_{\text {em }}$ of $405-430 \mathrm{~nm}$ in its fluorescent emission spectrum. As expected, the peaks around $405-430 \mathrm{~nm}$ were also presented in fluorescence spectra of DA-PLMA and DA-PLMA-PMAA, indicating that the fluorescence property was ascribed to the existence of rosin. The peak intensity of $405-430 \mathrm{~nm}$ was decreased for DA-PLMA but increased significantly for DAPLMA-PMAA, which was consistent with that of the UV/vis analysis. Overall, the results from both the UV/vis and fluorescent analysis confirm the existence of DA before and after acid hydrolysis, and DA-PLMA-PMAA showed the selffluorescence property which has promising application in the drug delivery field. It was normal that the Mn of DA-PLMAPMAA $(18,000 \mathrm{~mol} / \mathrm{g})$ was lower than that of DA-PLMA $(25,000 \mathrm{~mol} / \mathrm{g})$ after hydrolysis. However, after hydrolysis, the PDI of DA-PLMA-PMAA (after hydrolysis) was still as low as 1.17 (Figure 5B), which meant the partial hydrolysis process was reliable for the following preparation of micelles.

\section{DA-PLMA-PMAA Micelles With pH Sensitivity and Self-Fluorescence}

After acid hydrolysis of DA-PLMA copolymer, the resulting products contain both hydrophilic poly methacrylic acid and hydrophobic poly lauryl methacrylate which were commonly known to self-assemble to form micelle structures in aqueous solution. Indeed, the THF solution of DA-PLMA-PMAA was added into deionized water at room temperature, and the mixture was stirred for $24 \mathrm{~h}$ for the evaporation of THF. The morphology of micelles was observed using transmission electron microscopy (TEM). Figure 6A (inset) showed the TEM image of DA-PLMAPMAA in deionized water $(\mathrm{pH}=7)$ with concentration of 
$0.1 \mathrm{mg} / \mathrm{ml}$, from which the spherical micelles with the diameter of $\sim 40 \mathrm{~nm}$ were clearly seen. The hydrodynamic diameter (Z-average) of micelles obtained from DLS was $\sim 65 \mathrm{~nm}$, and the PDI of micelles was as low as 0.105 , which was much lower than that of sustainable micelles derived from chitosan or lignin (Zhou et al., 2019; Pornpitchanarong et al., 2020) and polymeric micelles prepared by Reversible Addition-Fragmentation Transfer (RAFT) polymerization (Wang C. et al., 2020). This probably ascribes to the relatively low PDI (1.17) of the DAPLMA-PMAA copolymer. In addition, the zeta potential was $-17.3 \mathrm{mV}$, and the negative surface could facilitate drug loading and the delivery process (Yang et al., 2017).

Surface tension measurements were adopted to determine the critical micellar concentration, which is an important parameter to characterize the stability of polymeric micelles (Patist et al., 2000; Calvo et al., 2009). As shown in Figure 6A, the surface tension came to be a constant when the surface is saturated at a surface tension value of $\sim 61.8 \mathrm{mN} / \mathrm{m}$ in $\mathrm{pH}=7$. The critical micellar concentration (CMC) read from the point of intersection between the two linear lines in Figure 6A was $22.5 \mathrm{mg} / \mathrm{L}$, which was in appropriate concentration, allowing their use in body fluids (Wei et al., 2007). In addition, the CMC of DA-PLMAPMAA micelles was much lower than that of common surfactant sodium lauryl sulfate (CMC was $2.5 \mathrm{~g} / \mathrm{L}$ ), suggesting that DAPLMA-PMAA micelles could be stable enough to serve as a drug carrier for drug delivery.

$\mathrm{pH}$ sensitivity is approved to be one of the most efficient stimuli for drug delivery. In order to investigate the $\mathrm{pH}$ influence on the formation of DA-PLMA-PMAA micelles, the size, size distribution, and zeta potential of the micelles in different $\mathrm{pH}$ were analyzed by DLS. As shown in Figures 6B,C, the hydrodynamic diameter (Z-average) was decreased with the increase of $\mathrm{pH}$; inversely, PDI increased with the increase of pHs. Particularly, in acidic solutions $(\mathrm{pH}=2$ and 5), the $\mathrm{Z}$-average value was $\sim 80 \mathrm{~nm}$, while in basic solutions $(\mathrm{pH}=$ 10 and 12 ), the $Z$-average value decreased to $\sim 50 \mathrm{~nm}$. This is mainly caused by conformational change of PMAA parts in acidic and basic solutions. It is well known that PMAA chains could be protonated and presenting expanding conformation in the acidic condition; however, in the basic condition, PMAA chains adopt a compact conformation due to the deprotonation of PMAA parts (Xiong et al., 2011; Yuan et al., 2012; Nan et al., 2014). Besides, due to the protonation of PMAA parts at low $\mathrm{pH}$, there were many positive charges in solution, resulting in a positive zeta potential value $(7.53 \mathrm{mV}, \mathrm{pH}=2 ; 0.25 \mathrm{mV}, \mathrm{pH}=5)$. With increasing $\mathrm{pH}$, carboxylic groups $(-\mathrm{COOH})$ in PAA could be highly ionized, resulting in a negative zeta potential value of the micelles solution $(-33.2 \mathrm{mV}, \mathrm{pH}=10 ;-33.1 \mathrm{mV}, \mathrm{pH}=12)$. The change of the $\mathrm{Z}$-average value and the zeta potential value of micelles solution vs. $\mathrm{pH}$ confirmed the $\mathrm{pH}$-dependent properties of micelles. As described above, DA-PLMA-PMAA showed an obvious self-fluorescence property under the excitation of $365 \mathrm{~nm}$. The fluorescence property of DA-PLMA-PMAA micelles in water was also investigated, and the bright fluorescence was observed under a UV lamp (365 nm) (Figure 6D). It is well known that fluorescent property is highly desirable for both in vitro and in vivo applications of drug-loading micelles because it allows for easy tracking of the nanocarriers using microscopy (Chen et al., 2015). Therefore, the resulted micelles with good fluorescence property would find promising application in the field of drug delivery.

\section{DOX Loading and In Vitro Release Studies of DA-PLMA-PMAA Micelles}

It is well known that micelles with large size could facilitate the drug release rate; however, particles with size over $200 \mathrm{~nm}$ are easily cleared by the reticuloendothelial system (RES) in vivo (Maeda and Matsumura, 2011). Therefore, the size of DAPLMA-PMAA was optimized to be the Z-average value of $\sim 85 \mathrm{~nm}$ for the drug-loading experiment. Thereafter, hydrophobic doxorubicin (Dox), a popular model anticancer drug, was chosen as a model drug for fundamental studies of drug loading and releasing properties of DA-PLMA-PMAA micelles. The size and PDI for Dox-loaded micelles were increased to $\sim 95 \mathrm{~nm}$ and 0.084 (Figure 7A), indicating that Dox was successfully loaded into micelles. The UV-vis spectrum of Dox, free micelles, and Dox-loaded micelles in Figure 7A (inset) showed the presence of Dox characteristic absorption peak at $\sim 495 \mathrm{~nm}$ for Dox and Dox-loaded micelles samples. This further confirmed a successful entrapment of Dox into the micelles.

The drug-loading capacity and drug-loading efficiency of DOX were analyzed by the UV-vis spectrophotometer at $495 \mathrm{~nm}$, and the results are summarized in Figure 7B. The drug-loading capacity (LC) of DOX increased from $8.9 \mathrm{wt} \%$ to $16.0 \mathrm{wt} \%$, corresponding to different feeding Dox concentrations at $\mathrm{pH}=7.0$, while the drug-loading efficiencies (LEs) decreased from 17.35 to $7.80 \%$. Commonly, physical entrapment of drugs in polymeric micelles is driven by the hydrophobic interactions as well as the electrostatic interaction between drugs and the polymer micelles. The LC of DA-PLMA-PMAA micelles was superior to that of most reported Dox-loaded polymeric micelles prepared by ATRP or other polymerization methods (Table 1 ). It is probably the similarity of aromatic chemical structures between DA and Dox that contributes to the good LC property of these micelles. As reported by Cuong et al. (2012), the aromatic folic acid could improve the LC of PEG-PCL polymeric nanocarrier from 5.5 to $10.9 \%$. Overall, the results indicated the feasibility of DA-PLMA-PMAA micelles as drug carriers.

To mimic the physiological blood environment, the in vitro release profiles of Dox-loaded polymeric micelles were examined in vitro at $37^{\circ} \mathrm{C}$ via a dialysis method using PBS buffers at $\mathrm{pH} 7.4$ (Figure 7C). The results showed an initial burst release of Dox (within $12 \mathrm{~h}$ ), followed by a sustained and slow release over a prolonged time (until $120 \mathrm{~h}$ ). Around $24 \%$ of Dox was released within first $12 \mathrm{~h}$, which could be attributed to the diffusion of Dox located close to the surface of the micelles. However, the amount of Dox released after $120 \mathrm{~h}$ was only $30.6 \%$, indicating that DAPLMA-PMAA micelles were relatively stable under physiological conditions. This can reduce the loss of drug during circulation in the blood stream ( $\mathrm{pH} 7.4$ ), which was highly desirable for drug carriers. 

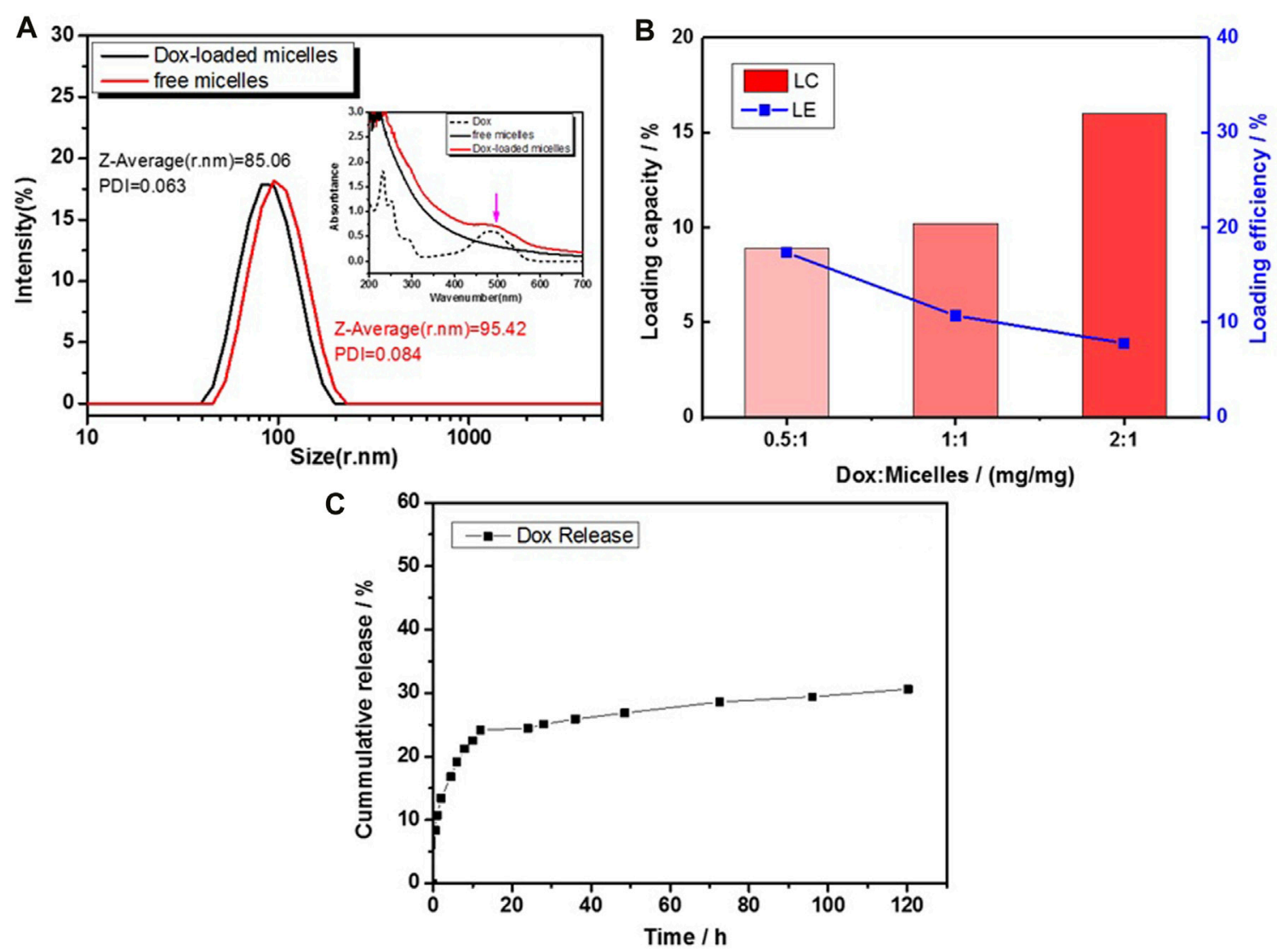

FIGURE 7 | (A) Size distribution by intensity (inset: UV-vis spectrum) for free micelles and Dox-loaded micelles; (B) drug-loading capacity and efficiency of Doxloaded micelles; and (C) release profile of Dox-loaded micelles in L in PBS (0.1 M, pH 7.4).

TABLE 1 | Drug-loading capacity (LC) of reported polymeric micelles.

\begin{tabular}{|c|c|c|c|c|c|}
\hline Entry & Polymer micelles & $\begin{array}{c}\text { Polymerization } \\
\text { method }\end{array}$ & Functional & LC (wt\%) & Reference \\
\hline 1 & PEG-b-PEYM & ATRP & $\mathrm{pH}$-sensitive & 2.6 & Tang et al. (2011) \\
\hline 2 & POEOMA nano-hydrogels & ATRP & - & $5.4-16.4$ & Oh et al. (2007) \\
\hline 3 & mPEG-PCL-PDMA/mPEG-PCL-PVBA-Dox & RAFT & $\mathrm{pH}$-sensitive & $8.1-10.1$ & Wang et al. (2020a) \\
\hline 4 & Folate-decorated star-shaped PEG-PCL & Free-radical polymerization & - & $4.6-13.0$ & Cuong et al. (2012) \\
\hline 5 & P(MPC-Co-PCL) & Free-radical polymerization & - & $6.7-12.6$ & Zhao et al. (2016) \\
\hline 6 & mPEG-b-P(HPMA-g-a-TOS-g-His) & Free-radical polymerization & $\mathrm{pH}$-sensitive & 9.6 & Debele et al. (2017) \\
\hline 7 & mPEG-PAsp(MEA) -PAsp(DIP) & Ring-opening polymerization & Reduction and $\mathrm{pH}$ sensitivity & 10.5 & Dai et al. (2011) \\
\hline 8 & H40-BPLP-PEG-OCH ${ }_{3} / \mathrm{CRGD}$ & Commercial PEG & Self-fluorescent and $\mathrm{pH}$-sensitive & 15.7 & Chen et al. (2015) \\
\hline 9 & LHRH-PEG-PHIS-Dox & Commercial PEG & pH-sensitive & 28 & Yang et al. (2015) \\
\hline 10 & DA-PLMA-PMAA & ATRP & Self-fluorescent and $\mathrm{pH}$-sensitive & $8.9-16.0$ & This study \\
\hline
\end{tabular}

\section{SUMMARY}

In summary, a facile strategy of preparation multifunctional polymeric micelles was successfully demonstrated. Sustainable dehydroabietic acid-based poly lauryl methacrylate (DAPLMA) with narrow PDI of 1.13 was prepared first via ATRP of hydrophobic lauryl methacrylate using rosin as an initiator. After simple acid hydrolysis, hydrophilic poly methacrylic acid (PMAA) moieties were formed, resulting in an amphiphilic polymer (DA-PLMA-PMAA) which spontaneously self-assembled into $\mathrm{pH}$-sensitive micelles in aqueous solutions. The spherical micelles had an average diameter of $\sim 65 \mathrm{~nm}$ and PDI as low as 0.105 , and showed self-fluorescence properties since they contained rosin segments. The Dox-loading capacity was as high as $16.0 \mathrm{wt} \%$, indicating that DA-PLMA-PMAA had a good 
drug-loading ability. The in vitro drug release study revealed that DA-PLMA-PMAA micelles were relatively stable under physiological conditions showing sustained-release characteristic. This study not only supported the DAPLMA-PMAA micelles as a promising efficient drug delivery carrier but also provided a facile approach to utilize renewable rosin and vegetable oils.

\section{DATA AVAILABILITY STATEMENT}

The original contributions presented in the study are included in the article/Supplementary Material; further inquiries can be directed to the corresponding authors.

\section{AUTHOR CONTRIBUTIONS}

JY: conceived and designed the experiments, performed experiments, analyzed the data, and contributed to original draft; CX and CL: performed experiments and analyzed the data; QL: analyzed the data; JW and FC: contributed to writing-review and editing.

\section{REFERENCES}

Bastakoti, B. P., Wu, K. C.-W., Inoue, M., Yusa, S.-i., Nakashima, K., and Yamauchi, Y. (2013). Multifunctional Core-Shell-Corona-Type Polymeric Micelles for Anticancer Drug-Delivery and Imaging. Chem. Eur. J. 19 (15), 4812-4817. doi:10.1002/chem.201203958

Biermann, U., Bornscheuer, U., Meier, M. A. R., Metzger, J. O., and Schäfer, H. J. (2011). "Oils and Fats as Renewable Raw Materials in Chemistry,"Angew. Chem. Int. Ed. 50, 3854-3871. doi:10.1002/anie.201002767

Cai, X., Lin, Y., Li, Y., Chen, X., Wang, Z., Zhao, X., et al. (2021). BioAIEgens Derived from Rosin: How Does Molecular Motion Affect Their Photophysical Processes in Solid State. Nat. Commun. 12 (1), 1-9. doi:10.1038/s41467-021-22061-y

Calvo, E., Bravo, R., Amigo, A., and Gracia-Fadrique, J. (2009). Dynamic Surface Tension, Critical Micelle Concentration, and Activity Coefficients of Aqueous Solutions of Nonyl Phenol Ethoxylates. Fluid Phase Equilibria 282 (1), 14-19. doi:10.1016/j.fluid.2009.04.016

Çayli, G., and Meier, M. A. R. (2008). Polymers from Renewable Resources: Bulk ATRP of Fatty Alcohol-Derived Methacrylates. Eur. J. Lipid Sci. Technol. 110 (9), 853-859. doi:10.1002/ejlt.200800028

Chen, G., Wang, L., Cordie, T., Vokoun, C., Eliceiri, K. W., and Gong, S. (2015). Multi-functional Self-Fluorescent Unimolecular Micelles for Tumor-Targeted Drug Delivery and Bioimaging. Biomaterials 47, 41-50. doi:10.1016/ j.biomaterials.2015.01.006

Cuong, N.-V., Li, Y.-L., and Hsieh, M.-F. (2012). Targeted Delivery of Doxorubicin to Human Breast Cancers by Folate-Decorated star-shaped PEG-PCL Micelle. J. Mater. Chem. 22 (3), 1006-1020. doi:10.1039/C1JM13588K

Dai, J., Lin, S., Cheng, D., Zou, S., and Shuai, X. (2011). Interlayer-Crosslinked Micelle with Partially Hydrated Core Showing Reduction and $\mathrm{pH}$ Dual Sensitivity for Pinpointed Intracellular Drug Release. Angew. Chem. Int. Ed. 50 (40), 9404-9408. doi:10.1002/anie.201103806

Debele, T. A., Lee, K.-Y., Hsu, N.-Y., Chiang, Y.-T., Yu, L.-Y., Shen, Y.-A., et al. (2017). A pH Sensitive Polymeric Micelle for Co-delivery of Doxorubicin and a-TOS for colon Cancer Therapy. J. Mater. Chem. B 5 (29), 5870-5880. doi:10.1039/c7tb01031a

Hattori, G., Hirai, Y., Sawamoto, M., and Terashima, T. (2017). Self-assembly of PEG/dodecyl-graft Amphiphilic Copolymers in Water: Consequences of the

\section{FUNDING}

This work was supported by the National Natural Science Foundation of China (grant number: 31901275), the Youth Science and Technology Innovation Fund of Nanjing Forestry University (grant number: CX2017006), the project funded by the National First-class Disciplines (PNFD), the project funded by the Priority Academic Program Development of Jiangsu Higher Education Institutions (PAPD), and the Overseas Research and Study Program of Jiangsu Universities.

\section{ACKNOWLEDGMENTS}

We would like to express our gratitude to the advanced analysis and testing center of Nanjing Forestry University for the assistance in this study.

\section{SUPPLEMENTARY MATERIAL}

The Supplementary Material for this article can be found online at: https://www.frontiersin.org/articles/10.3389/fbioe.2021.753808/ full\#supplementary-material

Monomer Sequence and Chain Flexibility on Uniform Micelles. Polym. Chem. 8 (46), 7248-7259. doi:10.1039/C7PY01719G

Hespel, L., Kaifas, E., Lecamp, L., Picton, L., Morandi, G., and Burel, F. (2012). Synthesis of $\mathrm{pH}-$ Sensitive Micelles from Linseed Oil Using Atom Transfer Radical Polymerisation (ATRP). Polymer 53 (20), 4344-4352. doi:10.1016/ j.polymer.2012.07.041

Maeda, H., and Matsumura, Y. (2011). EPR Effect Based Drug Design and Clinical Outlook for Enhanced Cancer Chemotherapy. Adv. Drug Deliv. Rev. 63 (3). doi:10.1016/j.addr.2010.05.001

Meier, M. A. R., Metzger, J. O., and Schubert, U. S. (2007). Plant Oil Renewable Resources as green Alternatives in Polymer Science. Chem. Soc. Rev. 36 (11), 1788-1802. doi:10.1039/B703294C

Nan, J., Chen, Y., Li, R., Wang, J., Liu, M., Wang, C., et al. (2014). Polymeric Hydrogel Nanocapsules: a Thermo and $\mathrm{pH}$ Dual-Responsive Carrier for Sustained Drug Release. Nano-micro Lett. 6 (3), 200-208. doi:10.1007/ BF03353784

Netopilı' 'k, M., and Kratochvı'1, P. (2003). Polystyrene-equivalent Molecular Weight versus True Molecular Weight in Size-Exclusion Chromatography. Polymer 44 (12), 3431-3436. doi:10.1016/S00323861(03)00258-1

Oh, J. K., Siegwart, D. J., Lee, H.-i., Sherwood, G., Peteanu, L., Hollinger, J. O., et al. (2007). Biodegradable Nanogels Prepared by Atom Transfer Radical Polymerization as Potential Drug Delivery Carriers: Synthesis, Biodegradation, In Vitro Release, and Bioconjugation. J. Am. Chem. Soc. 129 (18), 5939-5945. doi:10.1021/ja0691501

Patist, A., Bhagwat, S. S., Penfield, K. W., Aikens, P., and Shah, D. O. (2000). On the Measurement of Critical Micelle Concentrations of Pure and Technical-Grade Nonionic Surfactants. J. Surfact Deterg 3 (1), 53-58. doi:10.1007/s11743-0000113-4

Pornpitchanarong, C., Rojanarata, T., Opanasopit, P., Ngawhirunpat, T., and Patrojanasophon, P. (2020). Catechol-modified Chitosan/hyaluronic Acid Nanoparticles as a New Avenue for Local Delivery of Doxorubicin to Oral Cancer Cells. Colloids Surf. B: Biointerfaces 196, 111279. doi:10.1016/ j.colsurfb.2020.111279

Semen, J., and Lando, J. B. (1969). The Acid Hydrolysis of Isotactic and Syndiotactic Poly(methyl Methacrylate). Macromolecules 2 (6), 570-575. doi:10.1021/ma60012a003 
Sun, H., Zhang, Y., and Zhong, Z. (2018). Reduction-sensitive Polymeric Nanomedicines: An Emerging Multifunctional Platform for Targeted Cancer Therapy. Adv. Drug Deliv. Rev. 132, 16-32. doi:10.1016/ j.addr.2018.05.007

Sun, Y., Ma, Z., Xu, X., Liu, X., Liu, L., Huang, G., et al. (2020). Grafting Lignin with Bioderived Polyacrylates for Low-Cost, Ductile, and Fully Biobased Poly(lactic Acid) Composites. ACS Sustain. Chem. Eng. 8 (5), 2267-2276. doi:10.1021/ acssuschemeng.9b06593

Tang, R., Ji, W., Panus, D., Palumbo, R. N., and Wang, C. (2011). Block Copolymer Micelles with Acid-Labile Ortho Ester Side-Chains: Synthesis, Characterization, and Enhanced Drug Delivery to Human Glioma Cells. J. Controlled Release 151 (1), 18-27. doi:10.1016/j.jconrel.2010.12.005

Thyavihalli Girijappa, Y. G., Mavinkere Rangappa, S., Parameswaranpillai, J., and Siengchin, S. (2019). Natural Fibers as Sustainable and Renewable Resource for Development of Eco-Friendly Composites: a Comprehensive Review. Front. Mater. 6, 226. doi:10.3389/fmats.2019.00226

Tyrrell, Z. L., Shen, Y., and Radosz, M. (2010). Fabrication of Micellar Nanoparticles for Drug Delivery through the Self-Assembly of Block Copolymers. Prog. Polym. Sci. 35 (9), 1128-1143. doi:10.1016/ j.progpolymsci.2010.06.003

Wang, C., Qi, P., Lu, Y., Liu, L., Zhang, Y., Sheng, Q., et al. (2020a). Bicomponent Polymeric Micelles for pH-Controlled Delivery of Doxorubicin. Drug Deliv. 27 (1), 344-357. doi:10.1080/10717544.2020.1726526

Wang, J., Zhang, D., and Chu, F. (2020b). Wood-Derived Functional Polymeric Materials. Adv. Mater. 33 (28), 2001135. doi:10.1002/adma.202001135

Wang, Y., Kwak, Y., Buback, J., Buback, M., and Matyjaszewski, K. (2012). Determination of ATRP Equilibrium Constants under Polymerization Conditions. ACS Macro Lett. 1 (12), 1367-1370. doi:10.1021/mz3005378

Wei, H., Zhang, X., Cheng, C., Cheng, S.-X., and Zhuo, R.-X. (2007). Selfassembled, Thermosensitive Micelles of a star Block Copolymer Based on PMMA and PNIPAAm for Controlled Drug Delivery. Biomaterials 28 (1), 99-107. doi:10.1016/j.biomaterials.2006.08.030

Xiong, Z., Peng, B., Han, X., Peng, C., Liu, H., and Hu, Y. (2011). Dual-stimuli Responsive Behaviors of Diblock Polyampholyte PDMAEMA-B-PAA in Aqueous Solution. J. Colloid Interf. Sci. 356 (2), 557-565. doi:10.1016/ j.jcis.2011.01.067

Yang, S., Tang, Z., Zhang, D., Deng, M., and Chen, X. (2017). pH and Redox DualSensitive Polysaccharide Nanoparticles for the Efficient Delivery of Doxorubicin. Biomater. Sci. 5 (10), 2169-2178. doi:10.1039/c7bm00632b
Yang, T., Li, F., Zhang, H., Fan, L., Qiao, Y., Tan, G., et al. (2015). Multifunctional pH-Sensitive Micelles for Tumor-specific Uptake and Cellular Delivery. Polym. Chem. 6 (8), 1373-1382. doi:10.1039/C4PY01403K

Yu, J., Xu, C., Song, X., Lu, C., Wang, C., Wang, J., et al. (2021). Synthesis and Properties of Rosin Grafted Polymers Via "grafting from" ATRP: The Role of Rosin-Based Initiator. Ind. Crops Prod. 168, 113610. doi:10.1016/ j.indcrop.2021.113610

Yuan, W., Zhang, J., Zou, H., Shen, T., and Ren, J. (2012). Amphiphilic Ethyl Cellulose brush Polymers with Mono and Dual Side Chains: Facile Synthesis, Self-Assembly, and Tunable Temperature-pH Responsivities. Polymer 53 (4), 956-966. doi:10.1016/j.polymer.2012.01.003

Zhang, C., Garrison, T. F., Madbouly, S. A., and Kessler, M. R. (2017). Recent Advances in Vegetable Oil-Based Polymers and Their Composites. Prog. Polym. Sci. 71, 91-143. doi:10.1016/j.progpolymsci.2016.12.009

Zhao, Y., He, G., Guo, W., Bao, L., Yi, M., Gong, Y., et al. (2016). Self-assembled Micelles Prepared from Amphiphilic Copolymers Bearing Cell Outer Membrane Phosphorylcholine Zwitterions for a Potential Anti-phagocytic Clearance Carrier. Polym. Chem. 7 (36), 5698-5708. doi:10.1039/C6PY00845C

Zhou, Y., Han, Y., Li, G., Yang, S., Xiong, F., and Chu, F. (2019). Preparation of Targeted Lignin-Based Hollow Nanoparticles for the Delivery of Doxorubicin. Nanomaterials 9 (2), 188. doi:10.3390/nano9020188

Conflict of Interest: The authors declare that the research was conducted in the absence of any commercial or financial relationships that could be construed as a potential conflict of interest.

Publisher's Note: All claims expressed in this article are solely those of the authors and do not necessarily represent those of their affiliated organizations, or those of the publisher, the editors, and the reviewers. Any product that may be evaluated in this article, or claim that may be made by its manufacturer, is not guaranteed or endorsed by the publisher.

Copyright (c) $2021 \mathrm{Yu}, \mathrm{Xu}, \mathrm{Lu}$, Liu, Wang and Chu. This is an open-access article distributed under the terms of the Creative Commons Attribution License (CC BY). The use, distribution or reproduction in other forums is permitted, provided the original author(s) and the copyright owner(s) are credited and that the original publication in this journal is cited, in accordance with accepted academic practice. No use, distribution or reproduction is permitted which does not comply with these terms. 